\title{
Analysis of Vehicle Sperling Index Compared with the Flexible-rigid Coupled Model and Multi-body Rigid Model
}

\author{
Yijia Wang \\ Xi'an University of Science and Technology, Xi'an 710054, China \\ 648616394@qq.com
}

Keywords: Wheel our-of-roundness; Wear; Rigid-flexible coupling; Dynamic performance

\begin{abstract}
In order to understand the calculating difference between the multi-body rigid model and the flexible-rigid coupled model, especially the influence of the high frequency vibration caused by the wheel our-of-roundness(OOR) on sperling index and vibration acceleration response for standard wheel, polygon wheel and flat wheel are calculated and compared using the two models. The results show that the use of flexible-rigid coupled model not only inherits the achievement of the multi-body rigid model, but also to make up for the deficiency of multi-body rigid model in the study and can more accurately simulate the OOR wear caused by high frequency vibration on vehicle dynamic performance influence.
\end{abstract}

\section{Introduction}

At present, the research of railway vehicle dynamics in our country is mainly based on rigid body, which mainly stays on the criterion of sperling index and sperling index. But there are a lot of problems in the operation, which shows that the research of the vehicle system dynamics has some defects for the high-speed vehicles, and cannot meet the requirements. At present, vehicle dynamics is based on multi rigid body dynamics, ignoring the influence of elastic vibration, which affects the accuracy of calculation. Scholars at home and abroad consider the influence of a vehicle component on the dynamic performance of the system: Howell[1] analysis of vehicle vibration by taking the body as an elastic element of beam element; Suzuki [2,3] uses the variable stiffness Euler beam to simulate the body elastic vibration and study of the vehicle vibration and ride comfort caused by the track irregularity; Diana [4] used the modal superposition method to simulate the elasticity of the body and suspension, and studied the influence of vehicle elasticity and suspension system on the dynamic performance of high speed train; Yong [5] uses Timoshenko beam to simulate the vibration response of elastic body in bad lines; Eickhoff [6] is used to compare the difference between FE and element beam method; Netter [7] uses ANSYS to carry on the modal computation to the passenger car body, and introduces the result into SIMPACK to establish the corresponding rigid-soft coupling dynamic model. Zeng Jing [8] studied the influence of vehicle body elastic vibration on ride comfort in semi-active suspension vehicle system; Wu Pingbo [9] established a dynamic model of the bus system considering the elasticity of the vehicle body, and studied the effect of elastic vibration on the sperling index of the vehicle; Miao Bingrong [10] based on the combination of multi-body dynamics and finite element method of vehicle body structure fatigue simulation analysis. In this paper, unlike the previous studies, the vehicle body, the frame and the wheel are considered as the elastic body at the same time, and the corresponding rigid and flexible coupling model dynamic model is established. The sperling index index and the acceleration response of the two models under the three conditions (standard wheel, polygonal wheel and flat scar wheel) are compared, provide the basis for the definition of the scope of use of rigid flexible coupling dynamic model and the rigid body dynamics model.

\section{Vehicle System Dynamics Model}

The rigid body dynamics model considers the various parts of the vehicle system as a rigid body, ignoring the local vibration of each component, only considering the connection between the components. The rigid flexible coupling dynamic model has a unique dynamic effect, which can 
consider the coupling between the rigid body motion of the object and the elastic deformation of the component, as well as the unique dynamic effect caused by the coupling. The rigid flexible coupling model of the traditional, will only be a member of the vehicle system is considered as an elastic body, the other component is rigid, effects of high frequency vibration in order to more accurate study the wheel abrasion caused by the dynamic performance Of the vehicle body, frame, wheel parts considered as elastic body, then the combination a vehicle model of flexible construction. In the model, 600 nodes are considered in the car body, and the front and rear frames are all considered 200 nodes. The wheel set takes into account the total of 800 nodes. Fig. 1 shows the structure of the two kinds of dynamic models.

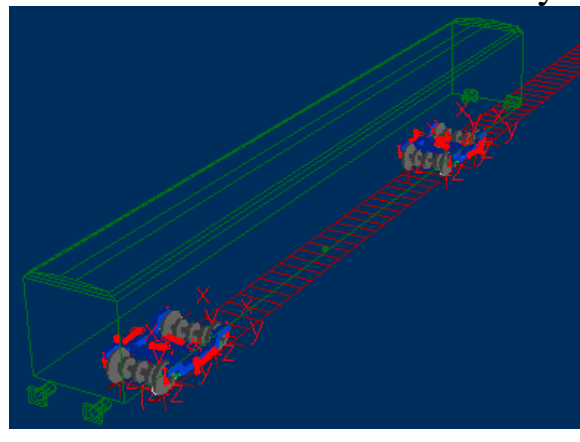

(a) Rigid body dynamics model

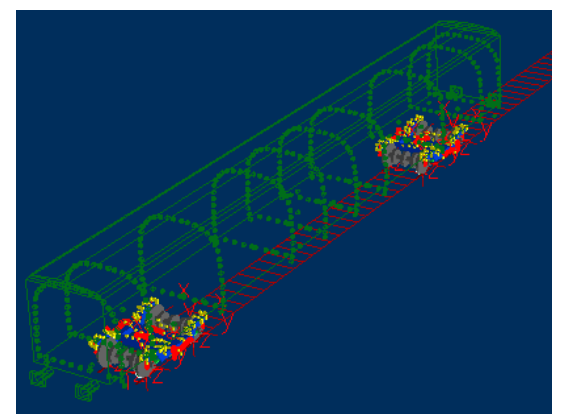

(b) Rigid Flexible Coupling Dynamics Model Figure 1. Rigid body model and rigid flexible coupling dynamic model

\section{Sperling Index}

For the simulation of the sperling index of the vehicle system, the track spectrum of the Beijing-Tianjin passenger dedicated line is used, in which the input of the track is the four directions irregularity.

Sperling Index of Standard Wheel. The vehicle sperling index of the standard wheel calculated by the rigid-flexible coupling dynamic model and the rigid body dynamics model is shown in Fig. 2 and Fig. 3. It can be seen from Fig. 2 that for the vertical sperling index index of the vehicle body, the rigid body dynamics model and the modulus value obtained by the rigid-flexible coupling dynamic model are consistent with the amplitude of the vehicle speed. When the vehicle speed is $250 \mathrm{~km} / \mathrm{h}$, The results of the rigid - flexible coupling model are larger than those of the rigid body model. This difference is more significant at high speed. It can be seen from Fig. 3, the vehicle lateral sperling index index, by using rigid model and rigid flexible coupling model calculation results differ only in the speed of $150 \mathrm{~km} / \mathrm{h}$ and $300 \mathrm{~km} / \mathrm{h}$, the calculation results of rigid flexible coupling dynamics model is slightly larger than the rigid model of the calculation results, the reason may be due to the elastic vibration the frequency resulted in weighting factor calculation sperling index index becomes large.
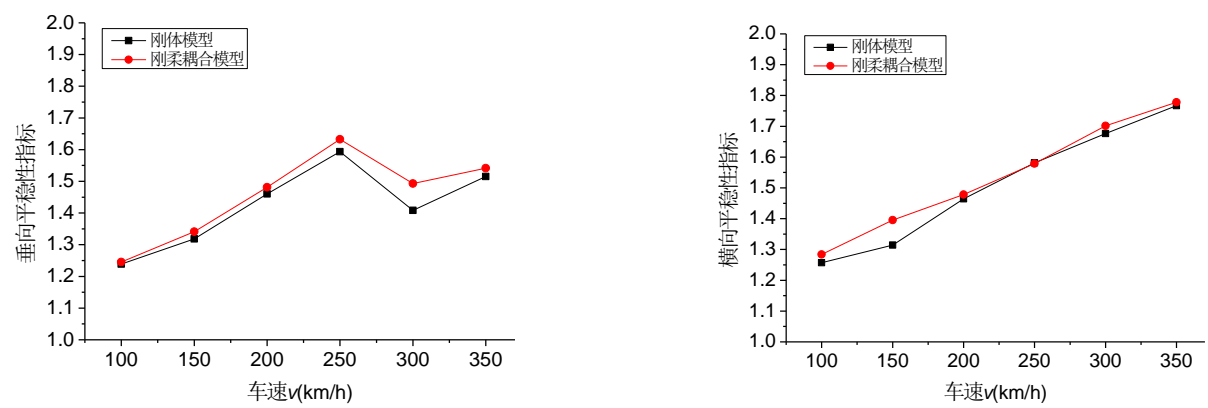

Figure 2. Comparison of vertical sperling index Figure 3. Comparison of transverse sperling index

Sperling Index of Polygon Wheel. Take the 3 order polygon as an example, the polygon amplitude is $0.1 \mathrm{~mm}$.

In Fig. 4 and Fig. 5, respectively, the rigid flexible coupling dynamic model and the rigid body dynamic model are used to calculate the vertical and lateral sperling index. Fig. 4 shows that the 
vertical sperling index of the rigid flexible coupling model is larger than that of the multi rigid body model. Fig. 5 shows that the vertical sperling index index calculated using the rigid-flexible coupling model is slightly larger than that of the multi-rigid body model, but the difference is not significant.
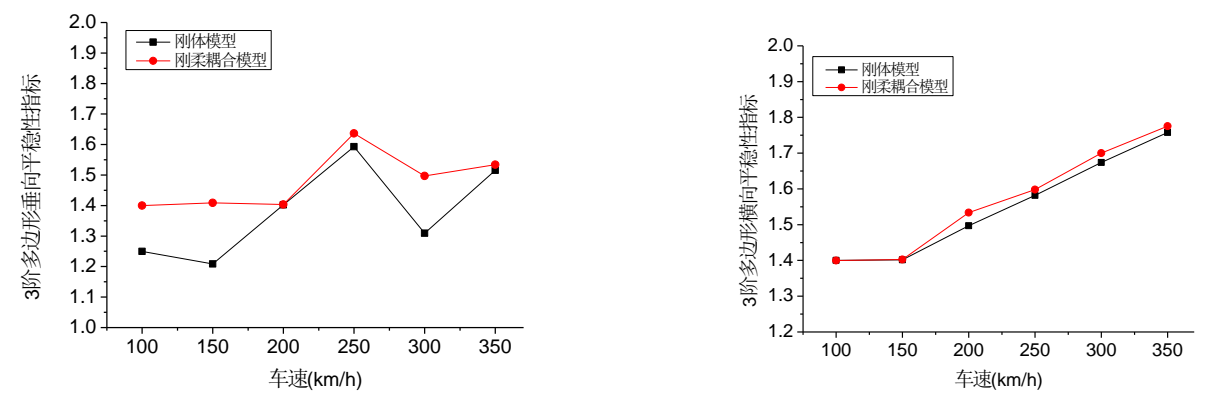

Figure 4. Comparison of vertical sperling index of third order polygon Figure 5 .Comparison of lateral sperling index of 3rd order polygons

Sperling Index of Wheel Flat. Taking the new flat model of the wheel as an example, the flat length $\mathrm{L}=20 \mathrm{~mm}$ is selected as the simulation object.

In Fig. 6 and Fig. 7, the vertical and lateral sperling index indexes are obtained by using the rigid flexible coupling dynamic model and the rigid body dynamics model. Fig. 6 shows that the vertical sperling index of the rigid flexible coupling model is much larger than that of the multi rigid body model. Fig. 7 shows that the vertical sperling index index calculated by the rigid flexible coupling model is larger than that of the multi rigid body model.
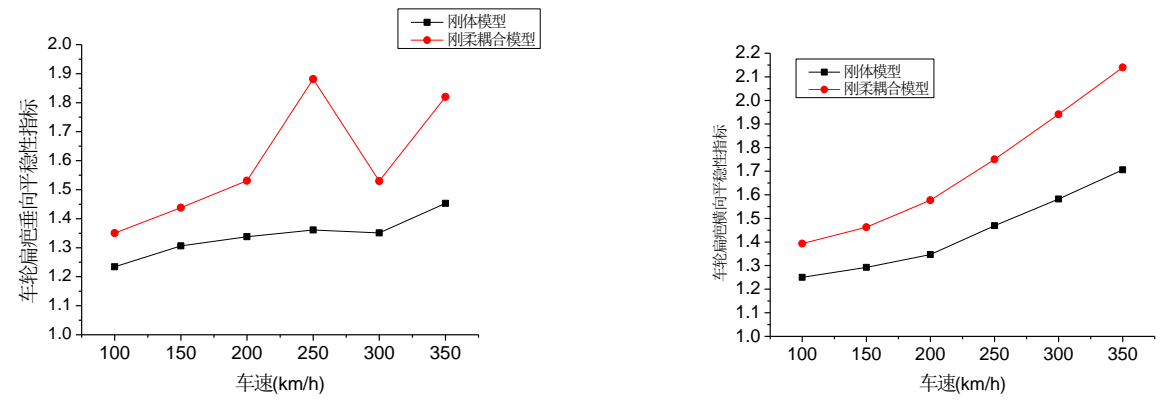

Figure 6. Comparison of vertical sperling index of flat wheel Figure 7. Comparison of vertical sperling index of flat wheel

\section{Vertical Vibration Acceleration Response}

Comparison of calculation standard wheels, polygonal wheel and wheel flat under the condition of rigid model and rigid flexible coupling model of the vehicle body, frame and wheelset vertical vibration acceleration response, mainly on the vertical vibration acceleration curve, the vertical vibration acceleration amplitude frequency and power density spectrum. The vehicle simulation speed is $300 \mathrm{~km} / \mathrm{h}$, and the track spectrum is the Beijing-Tianjin passenger line.

Vertical Vibration Acceleration Response of Standard Wheel. In Fig. 8 and Fig. 9 show the amplitude-frequency curves of the vertical acceleration in the middle of the vehicle body by the rigid-flexible coupling model and the vertical acceleration time curve calculated by the rigid body dynamics model at $300 \mathrm{~km} / \mathrm{h}$ operating speed. Fig. 10 and Fig. 11 show the acceleration curve and amplitude-frequency curve of the middle part of the vehicle body calculated by the rigid-flexible coupling dynamic model at the speed of $300 \mathrm{~km} / \mathrm{h}$. It can be seen from Fig. 8 and Fig. 10 that the acceleration of the vertical vibration of the body the Beijing-Tianjin line is obviously smaller than that calculated by the rigid-flexible coupling model. To the vibration acceleration of $0.2 \mathrm{~m} / \mathrm{s}^{2}$ or so, while the latter calculated the maximum vertical vibration acceleration of the body reached $0.7 \mathrm{~m} /$ $\mathrm{s}^{2}$, the former and the latter probably difference of nearly $70 \%$. It can be seen that the frequency amplitude of the rigid-flexible coupling dynamics model is much larger than that of the rigid body dynamics model because the rigid-flexible coupling dynamics model is in the elastic vibration and the rigid body mode have a lot of energy. $0.6866 \mathrm{~Hz}$ is the floating frequency, $15.73 \mathrm{~Hz}$ is the first 
order vertical bending frequency.

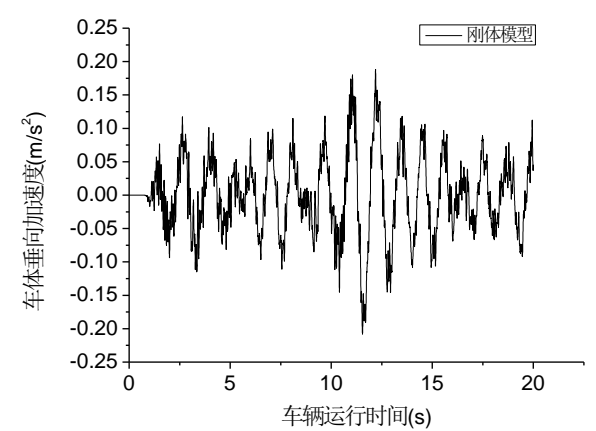

Figure 8. Vehicle acceleration time course curve

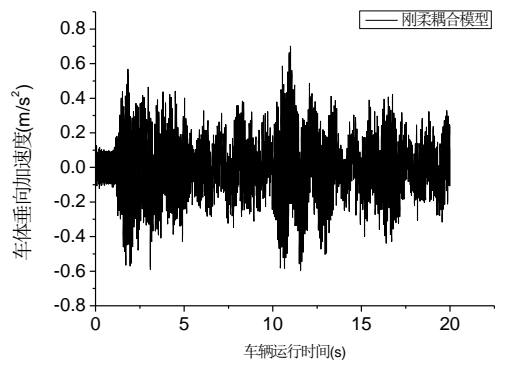

Figure 10. Vehicle acceleration time course curve

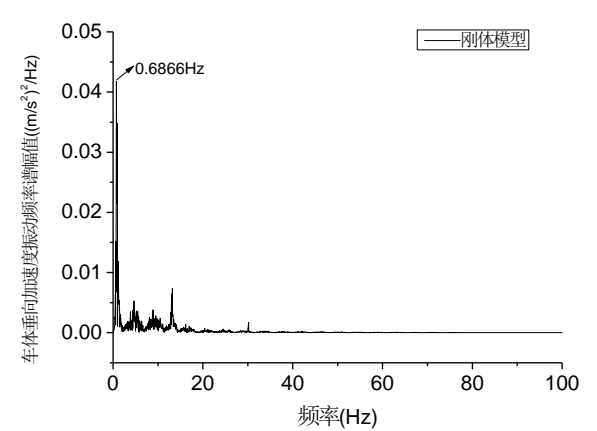

Figure 9. Body acceleration amplitude-frequency curvee

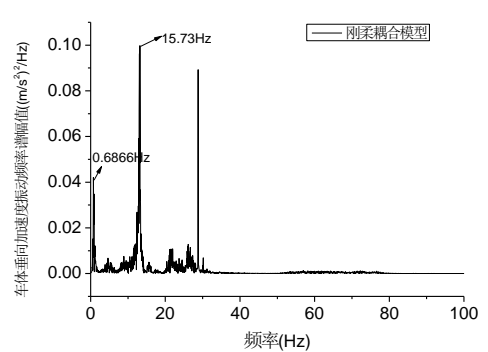

Figure 11. Body acceleration amplitude -frequency curve

Fig. 12 is the vibration acceleration power density calculated by rigid body dynamics model and rigid flexible coupling model, from the figure can be seen clearly that: in the low frequency stage, the two models of vertical vibration acceleration power spectral density is close to, but in the $15 \mathrm{~Hz}$ when the power density spectrum of the rigid flexible coupling model calculation results. The amplitude was significantly greater than that of the rigid body model in $15 \mathrm{~Hz}-100 \mathrm{~Hz}$. The calculation results of two kinds of model differences are more obvious. The reason is that the elastic vibration effect of the vehicle body is taken into account in the rigid- flexible coupling dynamic model, but the rigid body model is not considered.

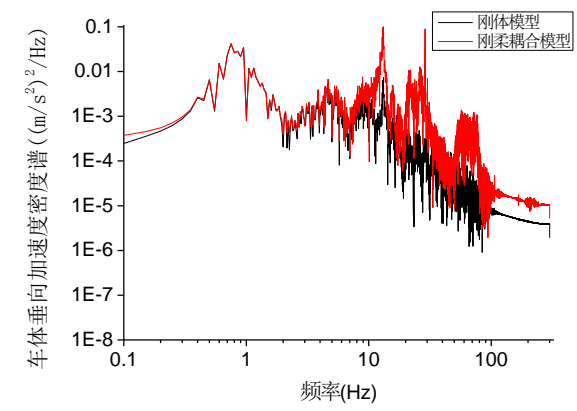

Figure 12. Vehicle body vibration acceleration power density spectrum

Vertical vibration acceleration response of wheel polygon. Take the 3 order polygon as an example, the polygon amplitude is $0.1 \mathrm{~mm}$.

Fig. 13 and Fig. 14 show the amplitude-frequency curves of the vertical acceleration in the middle of the vehicle body and the vertical acceleration time curve calculated by the rigid body dynamics model at $300 \mathrm{~km} / \mathrm{h}$ operating speed. Fig. 15 and Fig. 16 show the amplitude-frequency curves of the vertical acceleration in the middle of the vehicle body by the rigid-flexible coupling model and the vertical acceleration time curve calculated by the rigid-flexible coupling model at $300 \mathrm{~km} / \mathrm{h}$ operating speed. It can be seen from Fig. 13 and Fig. 15 that for the acceleration amplitude of the vertical vibration of the 3rd-order polygonal wheel, the result of the rigid body model calculated is obviously smaller than that calculated by the rigid-flexible coupling model. To the vibration acceleration of $0.2 \mathrm{~m} / \mathrm{s}^{2}$ or so, while the latter calculated the maximum vertical vibration acceleration of the body reached $0.7 \mathrm{~m} / \mathrm{s}^{2}$, the former and the latter probably difference of nearly $70 \%$. It can be seen from Fig. 14 and Fig. 16 that the frequency amplitude of the 
rigid-flexible coupling dynamics model is much larger than the rigid body dynamics model, especially for the vibration frequency caused by the third-order polygon, since the rigid-flexible coupling model has a lot of energy in elastic vibration and rigid body mode, while the rigid body model has only energy in rigid body mode. In Fig. 14, $0.6866 \mathrm{~Hz}$ is the floating frequency of the vehicle body, $15.73 \mathrm{~Hz}$ is the first order bending frequency of the elastic body, and $86.54 \mathrm{~Hz}$ is the vertical vibration frequency caused by the third order polygon.

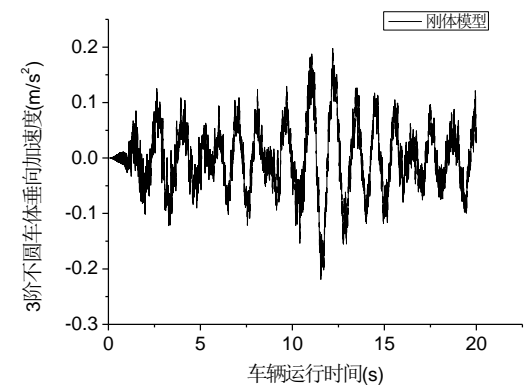

Figure 13. Vehicle acceleration time course curve of third-order polygon

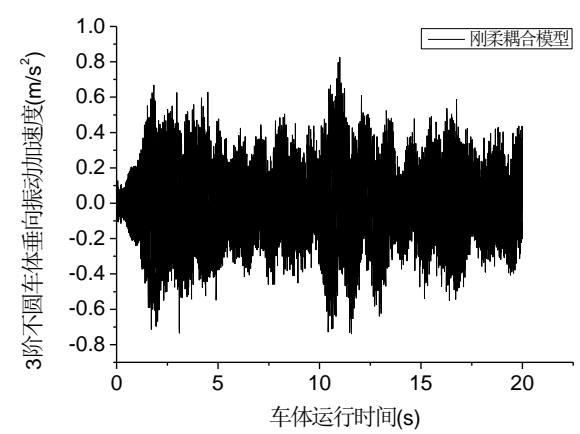

Figure 15. Vehicle acceleration time course curve of third-order polygon

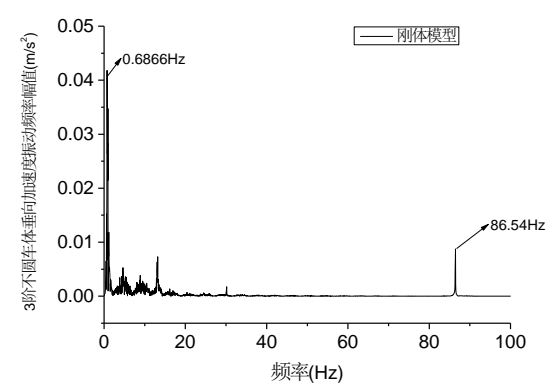

Figure 14. Body acceleration amplitude -frequency curve of third-order polygon

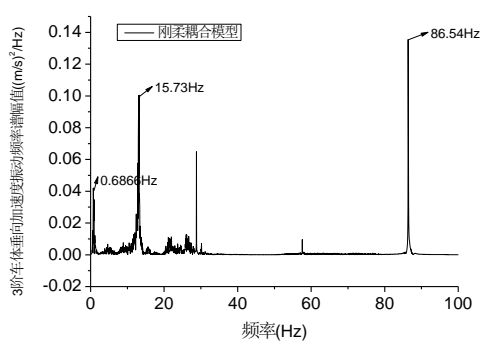

Figure 16. Body acceleration amp litude-frequency curve of third-order polygon

Fig. 17 is the vibration acceleration power density calculated by rigid body dynamics model and rigid flexible coupling model, from the figure can be seen clearly that: In the low frequency stage, the vertical vibration acceleration power spectral density of the two models is close, but the amplitude of the power density spectrum of the rigid-flexible coupling model is obviously larger than that of the rigid body model at $15 \mathrm{~Hz}$. the calculation of the two models The results are even more pronounced at $15 \mathrm{~Hz}-100 \mathrm{~Hz}$. The reason is that the elastic vibration effect of the vehicle body is taken into account in the rigid-flexible coupling dynamics model of the third-order polygon wheel due to the elastic vibration effect of the high-speed body, and the rigid body model is not considered.

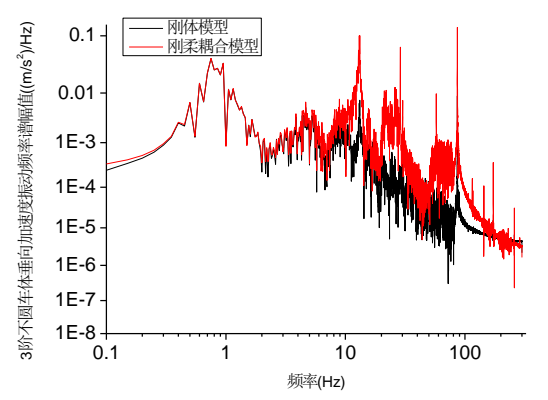

Figure 17. Vehicle body vibration acceleration power density spectrum of third-order polygon Vertical vibration acceleration response of wheel flat. New wheel flat model, flat scar length $\mathrm{L}=$ $20 \mathrm{~mm}$.

Fig. 18 and Fig. 19 show the amplitude-frequency curves of the vertical acceleration in the middle of the vehicle body and the vertical acceleration time curve calculated by the rigid body 
dynamics model at 300km / h operating speed. Fig. 20 and Fig. 21 show the amplitude-frequency curves of the vertical acceleration in the middle of the vehicle body and the vertical acceleration time curve calculated by the by the rigid-flexible coupling model at $300 \mathrm{~km} / \mathrm{h}$ operating speed. It can be seen from Fig. 18 and Fig. 20 that the acceleration of the vertical vibration acceleration of the body of the wheel flat on the Beijing-Tianjin line is much smaller than that calculated by the rigid-flexible coupling model. The maximum vertical vibration acceleration of the vehicle body is about $0.125 \mathrm{~m} / \mathrm{s}^{2}$, while the latter has the maximum vertical vibration acceleration of $0.55 \mathrm{~m} / \mathrm{s}^{2}$. The former and the latter are about 77\%. From the Fig. 19 and Fig. 21, the amplitude of the vertical vibration of the vehicle can be seen that the amplitude frequency of the rigid-flexible coupling model of the wheel flat is much larger than the rigid body, especially for the vibration frequency due to the impact, because the rigid-flexible coupling dynamics model in the elastic vibration and rigid body mode have a lot of energy, and rigid body model only in the rigid body mode energy. In Fig. $22,0.6866 \mathrm{~Hz}$ is the floating frequency of the vehicle body, $15.73 \mathrm{~Hz}$ is the first-order vertical bending frequency.

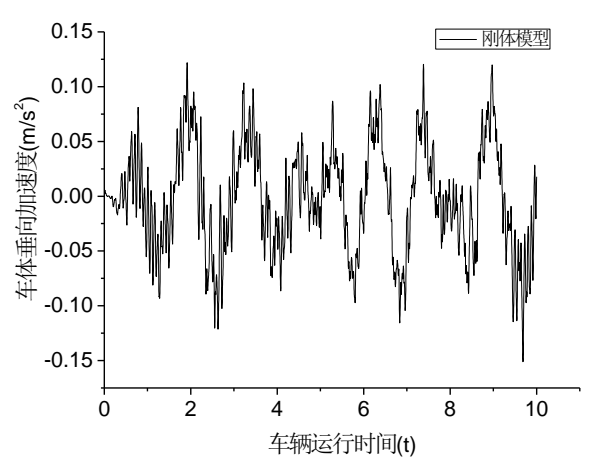

Figure 18. Vehicle acceleration time course curve of wheel flat

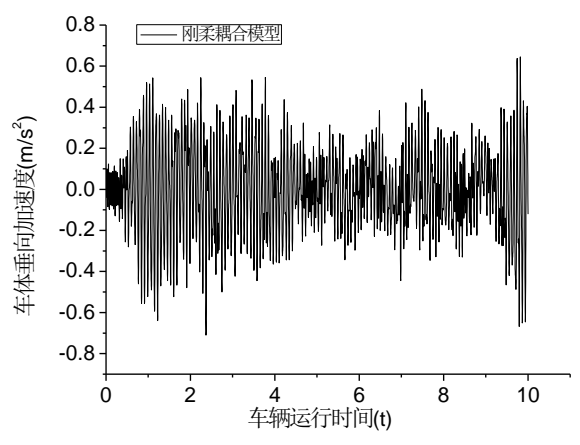

Figure 20. Vehic le acceleration time course curve of wheel flat

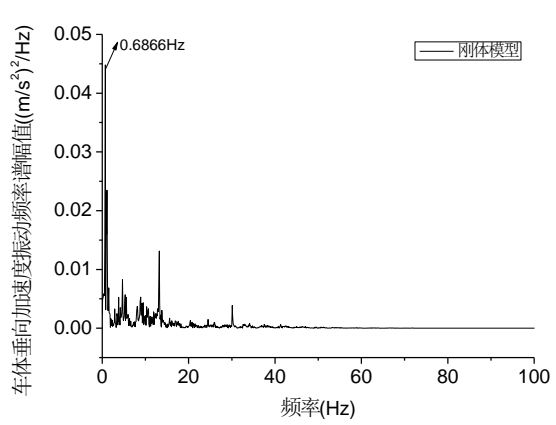

Figure 19. Body acceleration amplitude-frequency curve of wheel flat

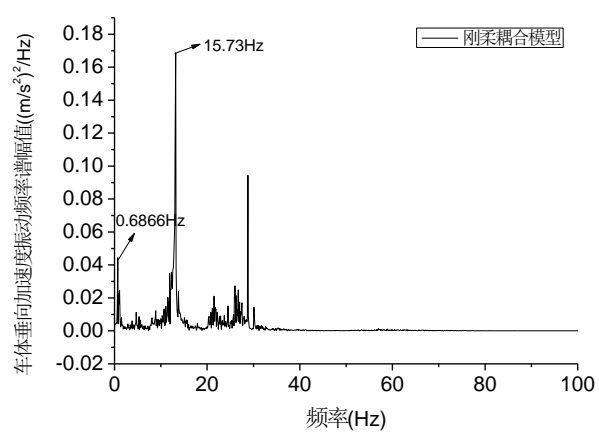

Figure 21. Body acceleration amplitude-frequency curve of wheel flat

Fig. 22 is the vibration acceleration power density calculated by rigid body dynamics model and rigid flexible coupling model, from the figure can be seen clearly that: In the low frequency stage, the spectral density of the vertical vibration acceleration of the two models is basically the same, but the amplitude of the power density spectrum of the rigid-flexible coupling models obviously larger than that of the rigid body model at $15 \mathrm{~Hz}$. The difference between the calculation results is more obvious at $15 \mathrm{~Hz}-100 \mathrm{~Hz}$. The reason is that the elastic vibration effect of the vehicle body is taken into account in the rigid-flexible coupling dynamic model, and the rigid body model is not considered. 


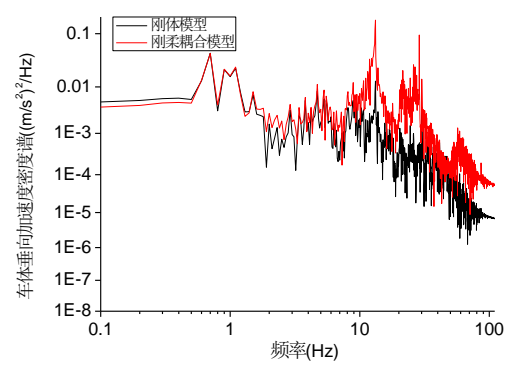

Figure 22. Vehicle body vibration acceleration power density spectrum of wheel flat

\section{Summary}

The sperling index of the rigid model and the rigid-flexible coupling model under the standard wheels, the wheel polygon and the wheel flat scar are compared:

For the sperling index of the vehicle: the vertical sperling index of the vehicle calculated by the rigid model and the rigid-flexible coupling model, whether the standard wheel or the third-order polygon and the wheel flat condition, the calculated result amplitude difference is large, The former is smaller than the latter, and with the speed increase, the difference is more obvious; However, the calculated results of the rigid-flexible coupling model and the rigid model are different in the third-order polygon and the wheel flat scar condition, but the calculation results of the two models are different. For the lateral sperling index index the results of the two models are not significant for the standard wheel condition, but the results of the rigid-flexible coupling model for the rigid body model are different in the case of the 3 order polygon and the wheel flat scar. Therefore, it is recommended to use a rigid-flexible coupling model when calculating the effect of out-of-round wheels.

The dynamic response of the body: Considering the elastic vibration of each component, both of the standard wheel and the third order polygon and the wheel flat scar condition, the rigid body model and the rigid and flexible coupling model calculated the vertical vibration acceleration response and frequency are very different. It is suggested that the influence of out-of-round wheels on the vibration of high - speed vehicle body is analyzed, and the rigid - flexible coupling model is used.

\section{Acknowledgements}

Project supported by Xi'an University of Science And Technology Training Fund Duration: 2014-2016, project approval number: 2014029.

Xi'an University of Science And Technology doctoral Foundation Fund Duration: 2015-2017, project approval number: 2015QDJ037.

61 China Postdoctoral Science Fund Duration: 2017/3-2018/9, project approval number: 2017M613292XB.

\section{References}

[1] L.J.Howell. Power spectral density analysis of vehicle vibration using the NASTRAN computer program[J].SAE Tran,1974, 83(2):1417-1424.

[2] Y.Suzuki and T. Tomioka. Method for flexible vibration damping of rolling stock carbody[J]. Railway Technical Research Institute, 1997,38(3):123-128.

[3] Y.Suzuki and K.A kutsu. Vibration analysis on truck frame of railway vehicle considering flexible rigidity[J]. Transaction of the Japan Society of Mechanical Engineers, 1997, 63(611):2221-2228. 
[4] G.Dinana, F. Cheli, S. Bruni and A. Collina. Dynamic interaction between rail vehicle and track for high speed train[J].Vehicle system dynamics ,1995,24(sup):15-30.

[5] T.H.Yong and C.Y.Li. Vertical Vibration analysis of vehicle/imperfect track system[J]. Vehicle system dynamics, 2003,40(5):329-349.

[6] B.M. Eickhoff and A.J.Minnis. A review of modeling methods for railway vehicle suspension components[J]. Vehicle system dynamics supplement, 1995,24(6-7): 469-496.

[7] Netter.H. New aspects of contact modeling and validation within multibody system simulation of railway vehicle[J]. Vehicle system dynamics,1998,28(sup):52-58.

[8] Zeng Jing, Luo Ren.Vibration Analysis of Railway Passenger Car Systems by Considering Flexible Car body Effect[J]. Journal of the china railway society,2007,29(6):19-25.

[9] Wu Pingbo, Xue Shihai, Yang Chenhui. Dynamic response of high-speed passenger car based on flexible car body model[J]. Journal of Traffic and Transport ation Engineering,2005,5(2):5-8.

[10] Miao Bingrong. Simulation research of locomotive carbody structure fatigue besed on multibody dynamics and finite element method[D]. Southwest Jiaotong University, Doctoral Dissertation,2003. 